\title{
Creation of Elite Rice With High-yield, Superior- quality and Strong Resistance to Brown Planthopper Based on Molecular Design
}

Manman Liu

Wuhan University

Fengfeng Fan

Wuhan University https://orcid.org/0000-0001-8942-3752

Shihao He

Wuhan University

Yu Guo

Wuhan University

\section{Gaili Chen}

Wuhan University

Nannan Li

Wuhan University

Nengwu Li

Wuhan University

Huanran Yuan

Wuhan University

Fengfeng Si

Wuhan University

\section{Fang Yang}

Wuhan University

Shaoqing Li ( $\nabla$ shaoqingli@whu.edu.cn )

Wuhan University https://orcid.org/0000-0002-6523-2158

\section{Original article}

Keywords: Molecular design breeding, Rice, High yield, Good quality, Brown planthopper

Posted Date: October 25th, 2021

DOI: https://doi.org/10.21203/rs.3.rs-992897/v1 
License: (c) (i) This work is licensed under a Creative Commons Attribution 4.0 International License. Read Full License 


\section{Abstract}

\section{Background}

Rice is the staple food feeding for more than half of the world's population. Breeding rice with high yield, superior quality and strong resistance is the goal of breeding to meet the needs of rapidly increased population. However, it is hard to achieve the aggregation of multiple complex traits based on traditional breeding methods.

Results

Here, we report the successful development of elite rice with high yield, superior quality and strong resistance to brown planthopper (BPH) by rational molecular design. According to the function of Gn8.1, APO1, Ghd7, Ghd8 and Gn1a for large panicle, Bph6 and Bph9 for brown planthopper, GW7, GS3 and qSW5 for grain appearance quality, and $W x, A L K, S B E 3, S S I V 2$ and SSIII for cooking and eating quality of rice, we staked all of the favorable genes together by crossing five donor lines one another. There are obvious phenotypic differences between different groups of the favorable genes. After careful screening of genotype, phenotype and genetic background, three molecular design lines were bred. The newly bred line exhibited higher yield potential, better grain quality and stronger BPH-resistance than their parents.

\section{Conclusions}

The successful creation of elite rice with high yield, superior quality and strong resistance to BPH demonstrate that molecular design at the basis of characterized genes is an effective and powerful strategy to improve multiple complex traits, it will provide a reference for the future commercial rice improvement.

\section{Background}

Rice (Oryza sativa L.), one of the most important staple grains in China, feeds more than $60 \%$ of the country's population (Cheng et al. 2007). To meet the demands of a growing population, rice production needs to be constantly increased (Tilman et al. 2011). Therefore, high yield is an eternal theme in rice breeding. However, with the continuous improvement of living standards, rice quality and function on human health are more cared by people. Recently, rice varieties with poor appearance, cooking and eating quality are no more welcomed by consumers in China (Zeng et al. 2017), which greatly limit their market value. Diseases and pests are the main enemies in rice production (Fan et al. 2017; Li et al. 2017), in order to reduce the harm of pests and diseases, a large amount of pesticides have to be applied in the rice production, which not only damage the environment but also cause pesticide residues in rice, leading to potential detriment to food safety (Zhang 2007). Developing varieties with strong resistance to insect pest, especially brown planthopper becomes a key manner to reduce the application of pesticide in rice production (Suh et al. 2013; Deng et al. 2017). Therefore, development of 'super-rice' varieties with high 
yield, good quality and strong resistance to insect pest is becoming a priority alternative in rice breeding programs.

In the last decades, a great progress has been achieved in rice breeding, but it is still difficult to meet the demand of consumption market (Qian et al. 2016). As the characters of high yield, good quality and strong resistance to pest and disease are hard to be efficiently integrated into elite rice using the traditional breeding methods (Fan et al. 2015; Liu et al. 2020). Recently, abundant work on markerassisted selection (MAS) has been performed to solve these problems, but successful cases are usually centered in improving traits of resistance to biological/abiotic stress, such as diseases, insects and drought, and rarely in complex traits such as grain yield and rice quality (Shamsudin et al. 2016; Wang et al. 2017; Ramalingam et al. 2020; Shailani et al. 2021). These phenomena are mainly owing to that most of the complex traits are controlled by quantitative loci with relatively low heritability and strong genotype-environment interaction, linkage block, and complex regulatory network (Zeng et al. 2017). Therefore, breeding of rice with high yield, superior quality and strong resistance is a considerable challenge for rice breeders because these characters are usually antagonistic to each other. Therefore, how to block or disrupt the potential conflict between grain yield, rice quality and resistance becomes a common concern of rice scientists.

With the rapid advances of plant functional genomics and deep understanding of complex trait regulation and molecular network, molecular design breeding, a new concept based on the extensive accumulated knowledge about the genes that regulate important agronomic traits was proposed (Qian et al. 2016), which greatly improve the accuracy and effectiveness of selection and accelerate the pyramiding of multiple complex traits as examplified by Zeng et al. (2017). An important prerequisite for molecular design breeding is the accumulation of genetic resources including favorable genes and important materials. Today, hundreds of quantitative trait loci (QTL) and gene for high grain yield, such as $A P 01, G h d 7$, Ghd8 and Gn1a, have been identified, but few of them have been widely employed in rice production (Ashikari et al. 2005; Ookawa et al. 2010; Zeng et al. 2017). In our previous research, we identified a high-yield gene, Gn8.1, from wild rice 0 . Iongistaminata, which can significantly increase grain number per panicle by $50 \%$, showing great application value in rice production (Fan et al. 2017). Rice quality is a complex trait controlled by at least 17 genes, of which, GW7, GS3 and qSW5 are selected as the target for grain appearance quality, and $W x, A L K, S B E 3, S S I V 2$ and SSIII as the target for eating and cooking quality (Wang et al. 2015; Zeng et al. 2017). The brown planthopper is a major harmful pest to rice production, today, more than $30 \mathrm{BPH}$-resistant genes having been genetically defined from wild and cultivated rice germplasms (Tan et al. 2020). Of which, Bph6 and Bph9 show strong resistance to the brown planthopper, and have great potential in rice breeding (Zhao et al. 2016; Guo et al. 2018). These important functional genes provide robust basis for us to optimize the grain yield, rice quality and resistance to brown planthopper as our design.

In order to improve these three complex characters of rice, we selected fifteen genes including Gn8.1 for large panicle in 0 . longistaminata BIL line 1880, GW7, GS3 and qSW5 for rice appearance quality, and $W x$, ALK, SBE3, SSIV2 and SSIII for cooking and eating quality in 9311 and Luo-Yu-Xiang (Basmati mutant), 
Bph6 and Bph9 for Bph-resistance in Luoyang6 and Luoyang9, respectively. After six years work, these 15 genes were pyramided together, and three elite rice lines showing high grain yield, superior quality and strong BPH-resistance were constructed as the goal of molecular design, which may provide a reference for the improvement of complicated traits of rice in the future.

\section{Results}

\section{Gene selection and phenotyping of the parental lines for molecular design}

The core of molecular design breeding is rational design, and our design strategy is shown as Additional file 1: Fig. S1. Firstly, according to the requirements of rice production and the needs of the market, we set the goals to develop high yield, superior quality and high BPH-resistant rice. We then selected fifteen genes including Gn8.1, GW7, GS3, qSW5, Wx, ALK, SBE3, SSIV2, SSIII, Bph6 and Bph9 as target genes to achieve this breeding goal (Fig. 1a). Then, we select appropriate donor rice with the corresponding genes for high yield, superior quality and strong BPH-resistance based on molecular markers (Fig. 1b). 9311, a well-known high-yield indica variety, was developed in China in 1997, and its hybrid Liang-You-Pei-Jiu was widely cultivated during the last decade (Zeng et al. 2017). Genotype identification showed that 9311 contains high-yield genes $A P O 1, G h d 7$, Ghd8 and Gn1a, appearance quality genes GS3 and $q S W 5$, eating and cooking quality genes $W x$ and $A L K$ (Additional file 1: Fig. S2). These excellent genes confer 9311 relatively high yield and fairly quality, although they show some space to the requirements of producers and consumers (Fig. 1C, Additional file 2: Table S1 and S2). Therefore, we used 9311 as the recurrent parent to further improve its yield, quality and BPH-resistance, which will be beneficial to the rapid realization of our breeding goals. 1880, a near isogenic line of 9311 carrying large panicle gene Gn8.1 from 0 . longistaminata, inherited all the excellent genes from 9311 except GS3 and ALK (Additional file 1: Fig. S2).

Compared with 9311, the rice quality of 1880 had no significant changes (Additional file 2: Table S2), but the number of grains per panicle increased by $68.4 \%$, which resulted in the yield per plant increased by $54.5 \%$ (Fig. 1c, Additional file 2: Table S1). Therefore, 1880 was selected as the donor parent for the large panicle. Luoyang- 6 and Luoyang-9, chromosome segment substitution lines carrying BPH-resistant genes Bph6 and Bph9 in 9311 genetic backgrounds, respectively, were used as the donor parents for $\mathrm{BPH}-$ resistance (Additional file 1: Fig. S3). Luo-Yu-Xiang, a superior quality rice derived from Basmati (Fig. 1C, Additional file 2: Table S2), was used as donor parent for quality genes SBE3, SSIV2, SSIII and GW7 (Additional file 1: Fig. S2). Finally, five donor parents including 9311, Luo-Yu-Xiang (LYX), 1880, Luoyang6 (LY6) and Luoyang-9 (LY9) were selected for pyramiding of the favorable target genes.

\section{Development of new rice lines with high-yield, superior- quality and BPH-resistance}


After determined of the parental rice lines, pyramiding of the target genes was carried out by crossing of the parents each other as shown in Figure 2. We firstly aggregated the excellent genes in 9311, 1880, LY6 and LY9, because these four parents have similar genetic backgrounds, which can accelerate achievement of the breeding goal of high yield and BPH-resistance. These four parents covered 11 target genes (Gn8.1, APO1, Ghd7, Ghd8, Gn1a, Wx, ALK, qSW5, GS3, Bph6 and Bph9), among which AP01, Ghd7, Ghd8, Gn1a, Wx and qSW5 were all included in the four parents (Additional file 1: Fig. S2), so we only need to screen Gn8.1, ALK, GS3, Bph6 and Bph9. During the breeding process, foreground selection was performed from the $\mathrm{F}_{1}$ to $\mathrm{BC}_{3} \mathrm{~F}_{4}$ generations and only progenies carrying all of the target alleles were selected for backcross or selfing. In detail, ten positive $F_{1}$ plants with all the target genes were identified and genotyped using the molecular markers from a $~ 300 \mathrm{~F}_{1}$ population derived from intercross of 1880/LY9//LY6, then, three selected plants were further backcrossed as maternal parents with 9311. In the $\mathrm{BC}_{1}$ generation, 13 out of 580 plants were identified as our expected candidates after careful genotyping and phenotyping. Similarly, the selected $\mathrm{BC}_{1}, \mathrm{BC}_{2}$ plants were then backcrossed with 9311 to eliminate the unexpected background. Then, $18 \mathrm{BC}_{3} \mathrm{~F}_{1}$ plants were selected for selfing until genetically stable in $\mathrm{BC}_{3} \mathrm{~F}_{4}$. Finally, three stable $\mathrm{BC}_{3} \mathrm{~F}_{4}$ lines with target genes were developed from 2700 plants (Fig. 2).

These three $\mathrm{BC}_{3} \mathrm{~F}_{4}$ lines successfully gathered all 11 target genes (Additional file 1: Fig. S4), and their phenotypes met the breeding goals of high yield and high BPH-resistant (Additional file 1: Fig. S5). Compared with 9311 , the grain numbers per panicle of the three $\mathrm{BC}_{3} \mathrm{~F}_{4}$ lines was increased by $51.2 \%$, $65.4 \%$ and $52.5 \%$, respectively, resulting in the plant yield increased by $43.1 \%, 60.7 \%$ and $46.6 \%$, respectively (Additional file 2: Table S3). The resistance scores showed that the $\mathrm{BPH}$-resistance of the three $\mathrm{BC}_{3} \mathrm{~F}_{4}$ lines increased from 9.0 in 9311 to 2.9, 3.0 and 3.1, respectively, meaning the plants showing strong resistance (Additional file 1: Fig. S6). However, the quality of these lines was not significantly different from that of 9311 (Additional file 2: Table S4), which needs to be further improved. Therefore, next, a $\mathrm{BC}_{3} \mathrm{~F}_{4}$ line was selected as the receptor parent to cross with Luo-Yu-Xiang to introgress good quality-related genes. Detailedly, the $\mathrm{F}_{1}$ obtained from $\mathrm{BC}_{3} \mathrm{~F}_{4} / \mathrm{LYX}$ was continuously backcrossed with the selected $\mathrm{BC}_{3} \mathrm{~F}_{4}$ line again, and the backcrossed progenies were screened based on molecular markers and phenotypes, and then the selected superior lines were further self-crossed. Finally, 18 stable lines were obtained.

\section{Genetic effects of different target gene combinations}

In order to well use of the target genes in the molecular design breeding, we analyzed the genetic effects of different target gene combinations with parent 9311 as control. According to the aggregation of target genes, the 18 molecular design breeding lines were divided into six groups (Additional file 1: Fig. S7). All five yield-related target genes, Gn8.1, APO1, Ghd7, Ghd8 and Gn1a, were aggregated in six groups. These five target genes were all genes controlling grain number, so the number of grains per panicle in six groups was significantly increased compared with 9311 (Fig. 3a). However, the increase of yield per plant was much smaller than that of grain number per panicle, which was due to the differences in effective 
panicle, Spikelet fertility and 1000-grain weight among different groups (Fig. 3a, b and Additional file 1: Fig. S8). For this reason, the yield per plant varies greatly among different lines in the same group, requiring careful screening based on phenotypic data (Additional file 2: Table S5). All six groups were polymerized with two $\mathrm{BPH}$ resistance genes $\mathrm{Bph} 6$ and $\mathrm{Bph} 9$, and the $\mathrm{BPH}$ resistance of the six groups was significantly improved compared with 9311 (Fig. 3c).

The differences between the groups were reflected in the aggregation of quality-related genes $G W 7$, SSIV2, SBE3 and SSIII-1. Group 1 aggregated all the target quality genes, and the quality traits of group 1 were significantly improved compared with 9311 (Fig. 3). Group 2 lost a gene SBE3 that regulates gel consistency and alkali spreading value (Tian et al. 2009), but the deletion of SBE3 did not result in significant changes in gel consistency and alkali spreading value compared with group 1 (Fig. $3 \mathrm{~g}$, i and Additional file 1: S7). This indicates that SBE3 is a small effect gene. Group 3 lost the gene $S S I V-2$, a gene that regulates alkali spreading value (Tian et al. 2009). The loss of this gene resulted in no significant improvement in alkali spreading value in group 3, which was consistent with 9311 but significantly decreased compared with the superior parent (Fig. 3i). Groups 5 and 6 were also missing SSIV-2 and showed low alkali spreading value like group 3 (Fig. 3i, Additional file 1: Fig. S7). This indicates that SSIV2 has a significant genetic effect on alkali spreading value regulation. The deletion of GW7, a gene that regulates the ratio of length to width (Wang et al. 2015), resulted in significantly lower length-width ratio in group 4 than in the non-deletion group (Such as Group 1/2/3) (Fig. 3e). Groups 5 and 6 were also missing GW7 and, like Group 4, also show a low length-width ratio (Fig. 3e, Additional file 1: Fig. S7). These results indicate that $G W 7$ has a significant genetic effect on regulating length-width ratio. In addition to the deletion of GW7 and SSIV-2, group 6 also lost one gene, SSIII-1, which regulates amylose content (Tian et al. 2009). However, the deletion of this gene did not result in significant differences in amylose content between groups (Fig. 3g). These results indicated that the genetic effect of SSIII-1 on amylose content was not obvious.

In general, the aggregation of a target gene shows an improvement in the corresponding target trait. However, we can also observe that different lines in the same group also show significant differences, which may be due to the hidden effect genes in the genetic background that we do not pay attention to. For example, the Group 1 including lines 01,13 and 14 contained all of the 15 target genes. The grain number per panicle of these three lines was significantly increased by $44.9 \%, 41.6 \%$ and $22.5 \%$ compared with 9311 , but the yield per plant was increased only by $22.9 \%, 10 \%$ and $18.2 \%$ due to the decrease of seed setting rate and 1000-grain weight (Additional file 2: Table S5). The BPH-resistance of these three lines reached to grade 4.7, 3.4 and 2.6, respectively (Additional file 1: Fig. S9). The quality performance of the three lines was significantly improved compared with that of 9311. In detail, the ratio of length/width, amylose content, gel consistency and alkali spreading value of line 01 were improved obviously, but the chalky grain rate was still as high as 9311 . The chalky grain rate of line 13 was not significantly reduced, and its alkali spreading value was obviously low. All the quality traits of line 14 were significantly improved, which were close to the level of superior-quality parent Luo-Yu-Xiang (Additional file 2: Table S6). Among these three lines, the performance of line 14 was particularly outstanding, and its yield, 
quality traits and resistance to brown planthopper were significantly improved. This indicates that careful phenotypic screening is an indispensable auxiliary means of molecular design breeding at present.

Comprehensively, the identified lines aggregated 14 or all of the favorable target gene, showed generally improved characters in BPH-resistance, grain yield and rice quality relative to 9311. Especially, the lines 07,08 and 14 showed apparently superior performance than their donor parents, and reached the target goals, and renamed as MD1 (Molecular design 1), MD2 and MD3, respectively.

\section{Genetic background profiling and phenotyping of the selected rice lines}

In the selected three elite rice lines based on molecular design, MD3 contained all 15 target genes, MD1 and MD2 collected all target genes except SBE3 (Fig. 4a). To determine whether the lines achieved the desired characters, we firstly investigated their yield characters (Fig. 4b-d and Table 1), the results showed that the MD1, MD2 and MD3 had excellent plant morphology, and inherited the characteristics of large panicle and high yield of 1880 (Fig. 4b), with obvious yield advantages to the other donor parents.

Relative to the Luo-Yu-Xiang, the grain yield of MD1, MD2 and MD3 increased about $56.8 \%, 47.6 \%$ and $55.9 \%$, respectively; compared with the recurrent parent 9311 , the grain yield increased about $21.7 \%$, $14.6 \%$ and $21.0 \%$, respectively (Table 1). Then, we investigated the grain quality of the three lines (Fig. 5), results showed that the lines had perfectly inherited the superior-quality characteristics of Luo-Yu-Xiang, and the appearance quality and cooking quality had been improved obviously. In detail, grain length and length/width ratio of the three lines were significantly higher than that of 9311 , but slightly lower than that of Luo-Yu-Xiang. In terms of chalky grain rate, the three lines perfectly inherited the low chalkiness of Luo-Yu-Xiang, which was significantly improved compared with 9311. In terms of amylose content, gel consistency and alkali spreading value, the three lines were significantly improved compared with 9311, and reached the high-quality level of the superior-quality parent Luo-Yu-Xiang (Fig. 5 and Table 2). Thirdly, we analyzed the resistance levels of brown planthopper in the three lines and their parents. The results showed that the BPH-resistant of the three lines MD1, MD2 and MD3 were significantly improved compared with the sensitive control 9311 (Fig. 6). The resistance scores of MD1, MD2 and MD3 were 3.2, 2.9 and 2.6, respectively, which were similar to that of LY69 (2.7), but significantly stronger than that of 9311 (9.0) (Fig. 6b). 
Table 1

Agronomic traits of selected molecular design lines and their parents in the design breeding.

\begin{tabular}{|c|c|c|c|c|c|c|c|}
\hline Lines & $\mathrm{PH}(\mathrm{cm})$ & PN & NGP & SF (\%) & GW (g) & YP (g) & GY (kg/plot) \\
\hline 9311 & $123 \pm 6^{a b}$ & $7.0 \pm 1.0^{b}$ & $171 \pm 6^{d}$ & $90.2 \pm 0.8^{a}$ & $29.2 \pm 0.2^{a}$ & $31.3 \pm 3.3^{c}$ & $2.95 \pm 0.37^{c}$ \\
\hline 1880 & $126 \pm 5^{a b}$ & $7.0 \pm 1.0^{b}$ & $288 \pm 8^{a}$ & $88.6 \pm 1.3^{\mathrm{ab}}$ & $29.4 \pm 0.4^{\mathrm{a}}$ & $52.1 \pm 6.5^{a}$ & $4.56 \pm 0.66^{a}$ \\
\hline LYX & $120 \pm 4^{b}$ & $8.2 \pm 0.3^{a}$ & $135 \pm 3^{e}$ & $87.3 \pm 1.8^{b}$ & $25.2 \pm 0.4^{c}$ & $24.4 \pm 1.3^{d}$ & $2.29 \pm 0.16^{d}$ \\
\hline MD1 & $127 \pm 8^{a}$ & $7.3 \pm 0.6^{b}$ & $227 \pm 12^{b c}$ & $88.3 \pm 1.1^{a b}$ & $27.7 \pm 0.7^{b}$ & $40.9 \pm 3.4^{b}$ & $3.79 \pm 0.40^{b}$ \\
\hline MD2 & $126 \pm 2^{\mathrm{a}}$ & $7.4 \pm 0.8^{b}$ & $231 \pm 14^{b}$ & $89.1 \pm 1.3^{\mathrm{ab}}$ & $24.6 \pm 1.1^{\mathrm{C}}$ & $37.6 \pm 1.3^{b}$ & $3.51 \pm 0.26^{b}$ \\
\hline MD3 & $123 \pm 7^{a b}$ & $8.1 \pm 0.2^{\mathrm{a}}$ & $210 \pm 15^{c}$ & $88.4 \pm 2.7^{a b}$ & $26.1 \pm 0.7^{b c}$ & $39.4 \pm 1.5^{b}$ & $3.67 \pm 0.28^{b}$ \\
\hline \multicolumn{8}{|c|}{$\begin{array}{l}\text { Note: PH, plant height; PN, panicle number; NGP, number of grains per panicle; SF, spikelet fertility; GW, } \\
1000-\text { grain weight; YP, yield per plant; Values are the means } \pm \text { s.d., } n=15 \text {. GY, Grain yield per plot; } \\
\text { means } \pm \text { s.d., } n=3 \text {. Letters indicate a significant difference at the } 5 \% \text { significance level by the least } \\
\text { significant difference test. }\end{array}$} \\
\hline
\end{tabular}

Table 2

The grain quality characters of parents and three molecular design breeding lines.

\begin{tabular}{|lllllll|}
\hline Materials & GL (cm) & L/W & CGR (\%) & AC (\%) & GC (mm) & ASV (GT) \\
\hline 9311 & $6.38 \pm 0.12^{\mathrm{d}}$ & $3.04 \pm 0.04^{\mathrm{e}}$ & $28.3 \pm 0.87^{\mathrm{a}}$ & $24.5 \pm 0.73^{\mathrm{a}}$ & $73.3 \pm 1.32^{\mathrm{c}}$ & $1.50 \pm 0.00^{\mathrm{b}}$ \\
\hline 1880 & $6.37 \pm 0.11^{\mathrm{d}}$ & $2.99 \pm 0.04^{\mathrm{e}}$ & $22.0 \pm 0.92^{\mathrm{b}}$ & $15.1 \pm 0.39^{\mathrm{c}}$ & $85.5 \pm 1.37^{\mathrm{b}}$ & $1.57 \pm 0.17^{\mathrm{b}}$ \\
\hline LYX & $7.13 \pm 0.17^{\mathrm{a}}$ & $3.98 \pm 0.06^{\mathrm{a}}$ & $4.7 \pm 0.35^{\mathrm{c}}$ & $16.5 \pm 0.33^{\mathrm{b}}$ & $86.7 \pm 1.51^{\mathrm{b}}$ & $6.03 \pm 0.32^{\mathrm{a}}$ \\
\hline MD1 & $6.65 \pm 0.12^{\mathrm{c}}$ & $3.41 \pm 0.09^{\mathrm{d}}$ & $4.6 \pm 0.22^{\mathrm{c}}$ & $16.7 \pm 0.39^{\mathrm{b}}$ & $90.3 \pm 1.35^{\mathrm{a}}$ & $6.02 \pm 0.28^{\mathrm{a}}$ \\
\hline MD2 & $6.70 \pm 0.13^{\mathrm{c}}$ & $3.63 \pm 0.05^{\mathrm{c}}$ & $4.3 \pm 0.25^{\mathrm{c}}$ & $17.1 \pm 0.42^{\mathrm{b}}$ & $85.5 \pm 1.27^{\mathrm{b}}$ & $6.17 \pm 0.33^{\mathrm{a}}$ \\
\hline MD3 & $6.93 \pm 0.16^{\mathrm{b}}$ & $3.85 \pm 0.06^{\mathrm{b}}$ & $3.5 \pm 0.18^{\mathrm{d}}$ & $15.7 \pm 0.45^{\mathrm{c}}$ & $85.3 \pm 1.37^{\mathrm{b}}$ & $6.01 \pm 0.39^{\mathrm{a}}$ \\
\hline $\begin{array}{l}\text { Note: GL, Grain length; L/W, Length-width ratio; CGR, Chalky grain rate; AC, Amylose content; GC, Gel } \\
\text { consistency; ASV, Alkali spreading value. Values are the means } \pm \text { s.d., n=15. Letters indicate a } \\
\text { significant difference at the 5\% significance level by the least significant difference test. }\end{array}$ & \\
\hline
\end{tabular}

Finally, in order to further identify the genetic background of the lines, 672 pairs of SSR primers evenly distributed on 12 chromosomes were selected for PCR identification. The lines of MD1, MD2 and MD3 had $85.0 \%, 82.5 \%$ and $82.4 \%$ of the genetic background of 9311 respectively (Additional file 2: Table S7). The others came from the parent of 1880, Luoyang 6, Luoyang 9 and Luo-Yu-Xiang; of which, the MD1, MD2 and MD3 harbored 23, 27 and 26 fragments from Luo-Yu-Xiang, apparently more than that from 1880, Luoyang 6 and Luoyang 9 (Additional file 1: Fig. S10), consistent with the fact that most of the genes for rice good quality come from Luo-Yu-Xiang. 
Combined with the excellent performance of the selected three lines in grain yield, rice quality, and resistance to brown planthopper, meaning that to synergistically improve multiple important agronomic traits such as resistance, yield and grain quality can be achieved in an optional molecular design.

\section{Discussion}

The eternal goal of rice breeding is to create super-varieties by pyramiding multiple desirable traits, such as high yield, superior quality, insect resistance and environmental stress tolerance into one variety. Molecular design breeding has unique advantages in achieving this goal, although less rice varieties have been bred since the concept was firstly proposed in 2003 (Peleman \& van der Voort 2003; lkeda et al. 2013). As rapid development of the function biology in recent years, more and more high-solution genomes and important functional genes for complex traits are easily accessed (Jiang et al. 2012; Li \& Zhang 2013; Huang et al. 2016), and the molecular design breeding will be greatly promoted. In this study, we firstly polymerize the high yield gene Gn8.1, brown planthopper resistant gene Bph6 and Bph9, then stake high-quality related genes with the Gn8.1, Bph6 and Bph9 together. There are two reasons to adopt this strategy. Firstly, the high yield line 1880, and brown planthopper resistant line Luoyang6 and Luoyang9 have similar genetic background with the recipient parent 9311, which is beneficial to the effective and rapid aggregation of the yield and resistance traits. Secondly, the grain yield trait is relatively more complex than the rice quality (Zeng et al. 2017), so it is relatively easy to improve the rice quality under high-yield background. This strategy facilitated us successfully to create elite rice with high yield, superior quality and high BPH-resistance within six years, a period much shorter than traditional breeding practice.

In our study, yield and resistance traits seems to be more easily clustered, this is owning to the large genetic effects and dominant characters of Gn8.1, Bph6 and Bph9. As long as a line harbors Gn8.1, Bph6 and $B p h 9$, it always displays high yield with large panicle and strong resistance to brown planthopper (Fig. 4 and 6, Additional file 1: Fig. S6 and S9, Additional file 2: Table S3 and S5), implying that these three genes have good genetic compatibility and great potentiality in rice molecular breeding. However, when yield, resistance and quality genes are clustered together, the agronomic traits of the lines become more complex. Among them, resistance traits were the most stable, followed by rice quality traits, and then the grain yield. In terms of resistance performance, although there were differences among 18 selected lines harboring $B P H 6$ and $B P H 9$, the $\mathrm{BPH}$-resistance level of all the lines was significantly improved compared with 9311 (Additional file 1: Fig. S9). In terms of quality traits, the quality traits can be significantly improved by polymerization of target genes related to rice quality. Certainly, there are also obvious phenotypic variations among different lines harboring the same target genes for a single quality index, because the selected genes for rice quality all showed minor genetic effects and tend to be affected by other undetected quality-related genes in the complex genetic background (Additional file 2: Table S6). In our study, GW7 and SSIV-2 showed strong genetic effects, indicating that these two genes have important application value in molecular design breeding. In terms of rice yield, the number of grains per panicle was significantly increased in the selected lines with all yield-related genes, but the yield per plant was significantly different (Additional file 2: Table S5). This is because the infiltration of 
high quality parental genetic background leads to the change of 1000-grain weight, seed-setting rate and panicle number, which leads to the different yield per plant. This further demonstrates the complexity of yield traits.

\section{Conclusions}

We have successfully created three new elite rice with high yield, superior quality and strong resistance to $\mathrm{BPH}$ by rational molecular design. The newly bred rice exhibited higher yield potential, better grain quality and stronger BPH-resistance than their parents. Our results demonstrate that molecular design is a powerful strategy to improve multiple complex traits, it will provide reference for the future commercial rice improvement.

\section{Materials And Methods Field experiment}

All of the rice materials were planted in a rice paddy fields during the standard growing season at the experimental field of Wuhan University in Hainan $\left(110^{\circ} 02^{\prime} \mathrm{E}, 18^{\circ} 48^{\prime} \mathrm{N}\right)$ and Ezhou $\left(114^{\circ} 35^{\prime} \mathrm{E}, 30^{\circ} 10^{\prime} \mathrm{N}\right)$ in 2015-2020. All seeds were soaked in water at $37^{\circ} \mathrm{C}$ in the dark for $24 \mathrm{~h}$ and were germinated at $37^{\circ} \mathrm{C}$ for $12 \mathrm{~h}$. The 25-day-old seedlings were transplanted in a ten-row plot with 12 plants per row by $20 \mathrm{~cm} \times 26$ $\mathrm{cm}$ spacing under a randomized complete block design with three replications. Standard field management was adopted with medium fertility level. Similar cultivation practices were performed each year.

\section{Investigation of agronomic traits in the field}

The measurements of the Plant height (PH), panicle number (PN), number of grains per panicle (NGP), spikelet fertility (SF), 1000-grain weight (GW) and yield per plant (YP) were performed at 30 days after heading. For all those traits, five representative plants in the middle of each repeat were chosen for trait measurement. Three replications were performed for each trait. The statistical analyses were performed with SPSS Statistics 20 (IBM, USA).

\section{Investigation of the grain quality}

One hundred fully filled shelled grains were randomly selected to determine grain length, grain width, length-to-width ratio and chalky kernels using the Wanshen SC-E type rice appearance quality detection analyzer (Hangzhou, China). The Amylose content (AC), Gel consistency (GC) and Alkali spreading value (ASV) were measured as previously described (Tian et al. 2009). All traits were measured with three replications.

\section{Evaluation of resistance to brown planthopper in rice materials}


The resistance of brown planthopper (BPH) in rice populations was evaluated by seedling identification, and the specific operation was based on previous described methods (Huang et al. 2001; Fan et al. 2017). Luoyang 69 (LY69), a line polymerized Bph6 and Bph9 genes with strong resistance to brown planthopper (Wang et al. 2017), was used as resistance control, while 9311 was used as susceptible control. When all of the seedlings of susceptible control died, the other rice lines were examined and each seedling was given a score of 1 to 9 according to the method of Huang et al. (2001). Each line was repeated three times, and the average of the three repetitions was the resistance score.

\section{Target gene screening and background profiling by molecular marker analysis}

Fifteen genes including Wx, ALK, SBE3, SSIV2, SSIII, GW7, GS3, qSW5, Gn8.1, APO1, Ghd7, Ghd8, Gn1a, $B p h 6$ and $B p h 9$, were selected as target genes, all of them were performed foreground selection in each generation of intercross and backcross using gene-specific PCR (Additional file 2: Table S8). A total of 258 SSR markers with polymorphism between the recurrent and donor parents (Additional file 2: Table S7) were used to identify the background of the derived descendent lines. The physical map was drawn using MapMaker Version 3.0 (Lander et al. 1987). The percentages of chromosome segments from recurrent parent in molecular design lines were reckoned as previously described (Xi et al. 2006; Suh et al. 2013).

\section{Abbreviations}

$\mathrm{BPH}$

brown planthopper

MAS

marker-assisted selection

QTL

quantitative trait loci

BIL

backcross inbred line

LYX

Luo-Yu-Xiang

LY6

Luoyang-6

LY9

Luoyang-9

MD

molecular design

$\mathrm{PH}$

plant height

PN 
panicle number

NGP

number of grains per panicle

SF

spikelet fertility

TGW

1000-grain weight

YP

yield per plant

$\mathrm{AC}$

amylose content, GC:gel consistency

ASV

alkali spreading value.

\section{Declarations}

\section{Ethical Approval and Consent to participate}

Not applicable.

\section{Consent for publication}

Not applicable.

\section{Availability of supporting data}

All data generated or analyzed during this study are included in this published article and its supplementary information files.

\section{Competing interests}

The authors declare that they have no competing interests.

\section{Funding}

This work was partly supported by the National Natural Science Foundation of China (U20A2023, 31870322), the Creative Research Groups of the Natural Science Foundation of Hubei Province (2020CFA009), and the Huanghe Patent Project of Wuhan City.

\section{Author contributions}

SL, FF and FY designed the research; ML, FF, SH, YG, GC, NL, NL, HY and FS performed field experiment and QTL analysis; ML, FF and SL analyzed data and wrote the manuscript. All authors read and approved the final manuscript. 


\section{Acknowledgments}

Not applicable.

\section{References}

Ashikari M., Sakakibara H., Lin S., Yamamoto T., Takashi T., Nishimura A., Angeles E.R., Qian Q., Kitano H. \& Matsuoka M. (2005) Cytokinin oxidase regulates rice grain production. Science 309, 741-745

Cheng S.H., Zhuang J.Y., Fan Y.Y., Du J.H. \& Cao L.Y. (2007) Progress in research and development on hybrid rice: a super-domesticate in China. Ann Bot 100, 959-966

Deng Y., Zhai K., Xie Z., Yang D., Zhu X., Liu J., Wang X., Qin P., Yang Y., Zhang G., Li Q., Zhang J., Wu S., Milazzo J., Mao B., Wang E., Xie H., Tharreau D. \& He Z. (2017) Epigenetic regulation of antagonistic receptors confers rice blast resistance with yield balance. Science 355, 962-965

Fan F., Li N., Chen Y., Liu X., Sun H., Wang J., He G., Zhu Y. \& Li S. (2017) Development of Elite BPHResistant Wide-Spectrum Restorer Lines for Three and Two Line Hybrid Rice. Front Plant Sci 8, 986

Fan F., Li N., Wang J., Liu X., Liu J., Zhu Y. \& Li S. (2015) Molecular marker-directed development of a novel cytoplasmic male sterile line in rice. Molecular Breeding 35, 212

Guo J., Xu C., Wu D., Zhao Y., Qiu Y., Wang X., Ouyang Y., Cai B., Liu X., Jing S., Shangguan X., Wang H., Ma Y., Hu L., Wu Y., Shi S., Wang W., Zhu L., Xu X., Chen R., Feng Y., Du B. \& He G. (2018) Bph6 encodes an exocyst-localized protein and confers broad resistance to planthoppers in rice. Nat Genet 50, 297-306

Huang X., Yang S., Gong J., Zhao Q., Feng Q., Zhan Q., Zhao Y., Li W., Cheng B., Xia J., Chen N., Huang T., Zhang L., Fan D., Chen J., Zhou C., Lu Y., Weng Q. \& Han B. (2016) Genomic architecture of heterosis for yield traits in rice. Nature $537,629-633$

Huang Z., He G., Shu L., Li X. \& Zhang Q. (2001) Identification and mapping of two brown planthopper resistance genes in rice. Theoretical and Applied Genetics 102, 929-934

Ikeda M., Miura K., Aya K., Kitano H. \& Matsuoka M. (2013) Genes offering the potential for designing yield-related traits in rice. Curr Opin Plant Biol 16, 213-220

Jiang Y., Cai Z., Xie W., Long T., Yu H. \& Zhang Q. (2012) Rice functional genomics research: progress and implications for crop genetic improvement. Biotechnol Adv 30, 1059-1070

Lander E.S., Green P., Abrahamson J., Barlow A., Daly M.J., Lincoln S.E. \& Newberg L.A. (1987) MAPMAKER: an interactive computer package for constructing primary genetic linkage maps of experimental and natural populations. Genomics 1, 174-181 
Li W., Zhu Z., Chern M., Yin J., Yang C., Ran L., Cheng M., He M., Wang K., Wang J., Zhou X., Zhu X., Chen Z., Wang J., Zhao W., Ma B., Qin P., Chen W., Wang Y., Liu J., Wang W., Wu X., Li P., Wang J., Zhu L., Li S. \& Chen X. (2017) A natural allele of a transcription factor in rice confers broad-spectrum blast resistance. Cell 170, 114-126

Li Z.K. \& Zhang F. (2013) Rice breeding in the post-genomics era: from concept to practice. Curr Opin Plant Biol 16, 261-269

Liu C., Ding S., Zhang A., Hong K., Jiang H., Yang S., Ruan B., Zhang B., Dong G., Guo L., Zeng D., Qian Q. \& Gao Z. (2020) Development of nutritious rice with high zinc/selenium and low cadmium in grains through QTL pyramiding. J Integr Plant Biol 62, 349-359

Ookawa T., Hobo T., Yano M., Murata K., Ando T., Miura H., Asano K., Ochiai Y., Ikeda M., Nishitani R., Ebitani T., Ozaki H., Angeles E.R., Hirasawa T. \& Matsuoka M. (2010) New approach for rice improvement using a pleiotropic QTL gene for lodging resistance and yield. Nat Commun 1, 132

Peleman J.D. \& van der Voort J.R. (2003) Breeding by design. Trends Plant Sci 8, 330-334

Qian Q., Guo L.B., Smith S.M. \& Li J.Y. (2016) Breeding high-yield superior quality hybrid super rice by rational design. National Science Review 3, 283-294

Ramalingam J., Raveendra C., Savitha P., Vidya V., Chaithra T.L., Velprabakaran S., Saraswathi R., Ramanathan A., Arumugam Pillai M.P., Arumugachamy S. \& Vanniarajan C. (2020) Gene pyramiding for achieving enhanced resistance to bacterial blight, blast, and sheath blight diseases in rice. Front Plant Sci 11,591457

Shailani A., Joshi R., Singla-Pareek S.L. \& Pareek A. (2021) Stacking for future: Pyramiding genes to improve drought and salinity tolerance in rice. Physiol Plant 172, 1352-1362

Shamsudin N.A., Swamy B.P., Ratnam W., Sta Cruz M.T., Raman A. \& Kumar A. (2016) Marker assisted pyramiding of drought yield QTLs into a popular Malaysian rice cultivar, MR219. BMC Genet 17, 30

Suh J.P., Jeung J.U., Noh T.H., Cho Y.C., Park S.H., Park H.S., Shin M.S., Kim C.K. \& Jena K.K. (2013) Development of breeding lines with three pyramided resistance genes that confer broad-spectrum bacterial blight resistance and their molecular analysis in rice. Rice (N Y) 6, 5

Tan J., Wu Y., Guo J., Li H., Zhu L., Chen R., He G. \& Du B. (2020) A combined microRNA and transcriptome analyses illuminates the resistance response of rice against brown planthopper. BMC Genomics 21, 144

Tian Z., Qian Q., Liu Q., Yan M., Liu X., Yan C., Liu G., Gao Z., Tang S., Zeng D., Wang Y., Yu J., Gu M. \& Li J. (2009) Allelic diversities in rice starch biosynthesis lead to a diverse array of rice eating and cooking qualities. Proc Natl Acad Sci U S A 106, 21760-21765 
Tilman D., Balzer C., Hill J. \& Befort B.L. (2011) Global food demand and the sustainable intensification of agriculture. Proc Natl Acad Sci U S A 108, 20260-20264

Wang S., Li S., Liu Q., Wu K., Zhang J., Wang S., Wang Y., Chen X., Zhang Y., Gao C., Wang F., Huang H. \& Fu X. (2015) The OsSPL16-GW7 regulatory module determines grain shape and simultaneously improves rice yield and grain quality. Nat Genet $47,949-954$

Wang Y., Jiang W., Liu H., Zeng Y., Du B., Zhu L., He G. \& Chen R. (2017) Marker assisted pyramiding of Bph6 and Bph9 into elite restorer line 93-11 and development of functional marker for Bph9. Rice (N Y) 10,51

Xi Z.Y., He F.H., Zeng R.Z., Zhang Z.M., Ding X.H., Li W.T. \& Zhang G.Q. (2006) Development of a wide population of chromosome single-segment substitution lines in the genetic background of an elite cultivar of rice (Oryza sativa L.). Genome 49, 476-484

Zeng D., Tian Z., Rao Y., Dong G., Yang Y., Huang L., Leng Y., Xu J., Sun C., Zhang G., Hu J., Zhu L., Gao Z., Hu X., Guo L., Xiong G., Wang Y., Li J. \& Qian Q. (2017) Rational design of high-yield and superior-quality rice. Nat Plants 3, 17031

Zhang Q. (2007) Strategies for developing Green Super Rice. Proc Natl Acad Sci U S A 104, 16402-16409

Zhao Y., Huang J., Wang Z., Jing S., Wang Y., Ouyang Y., Cai B., Xin X.F., Liu X., Zhang C., Pan Y., Ma R., Li Q., Jiang W., Zeng Y., Shangguan X., Wang H., Du B., Zhu L., Xu X., Feng Y.Q., He S.Y., Chen R., Zhang Q. \& He G. (2016) Allelic diversity in an NLR gene BPH9 enables rice to combat planthopper variation. Proc Natl Acad Sci U S A 113, 12850-12855

\section{Figures}




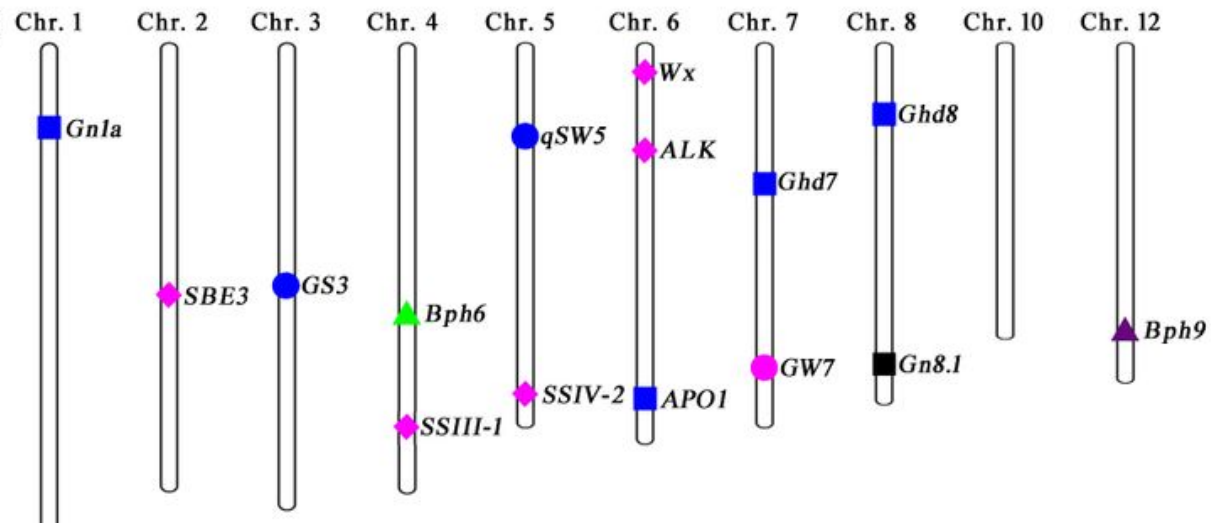

b

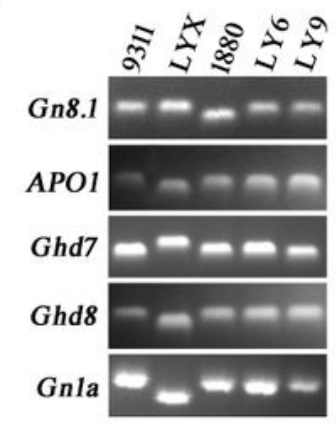

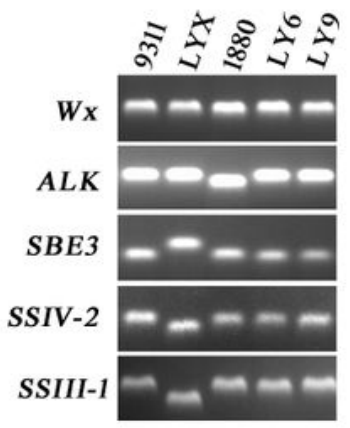

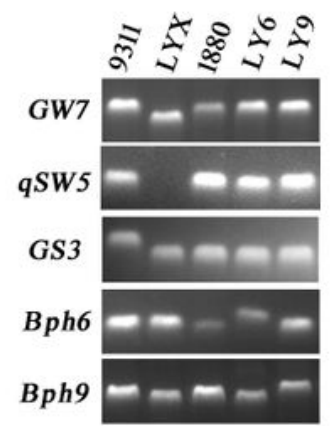

C
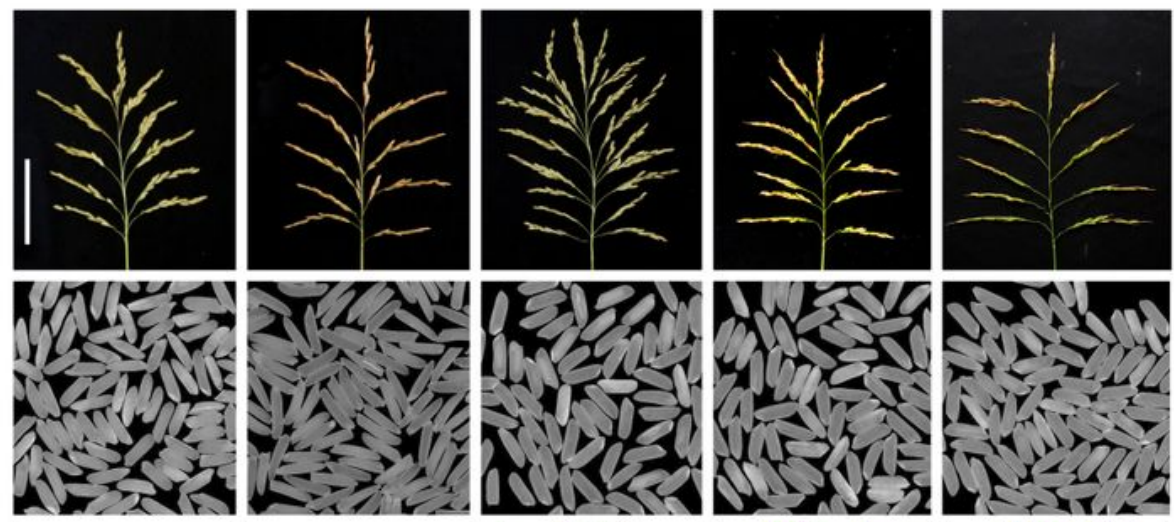

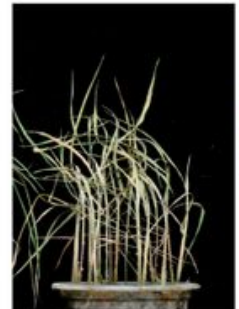

9311

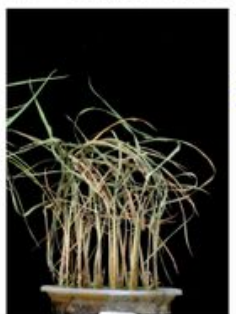

LYX

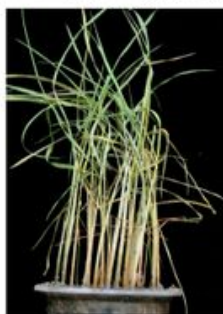

1880

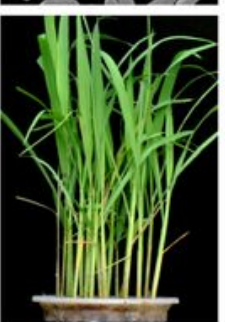

LY6
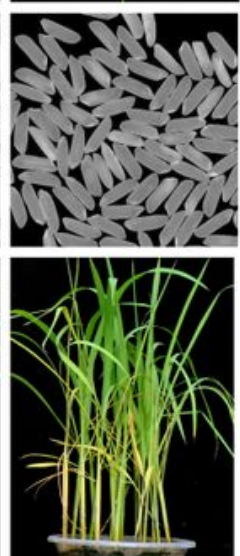

LY9

\section{Figure 1}

Description of target genes and parents selected for molecular design breeding. a: The distribution of target genes on chromosomes. Square represents yield-related genes, diamond represents eating and cooking quality related genes, circle represents appearance quality related genes, triangle represents rice $\mathrm{BPH}$-resistance related genes; Blue indicates that the favorable fragment is from 9311, pink indicates that the favorable fragment is from Luo-Yu-Xiang (LYX), black indicates that the favorable fragment is from 
1880, green indicates that the favorable fragment is from Luoyang 6 (LY6), and purple indicates that the favorable fragment is from Luoyang 9 (LY9). b: Polymorphism analysis of the target genes among parents 9311, LYX, 1880, LY6 and LY9. c: Yield, quality and resistance of five parents. At the top is the morphology of mature panicles, in the middle is the performance of the milled grain, and at the bottom is the resistance of brown planthopper at seedling stage.

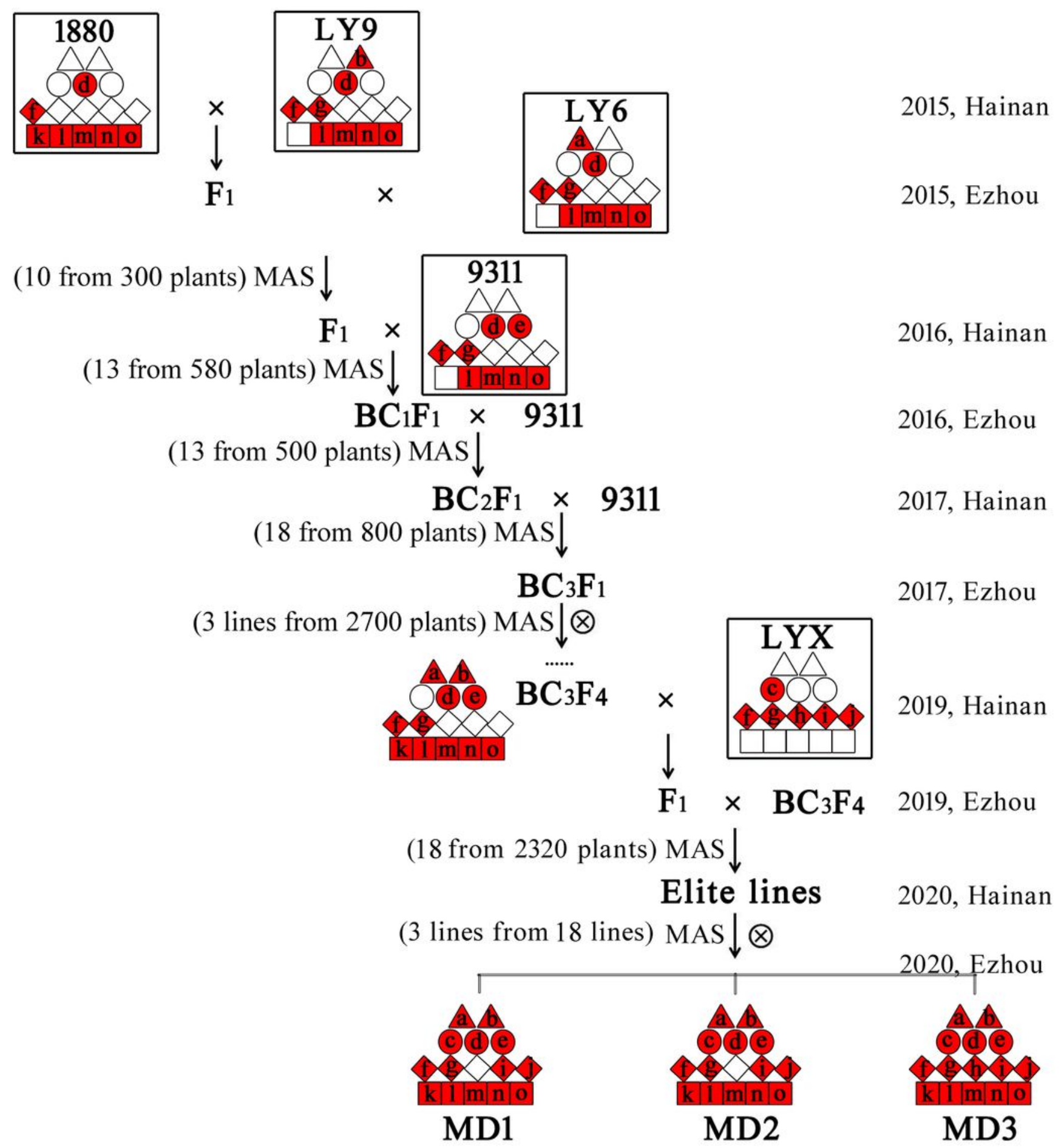

Figure 2 
Schematic diagram of molecular design breeding process. Square represents yield-related genes, in which k, I, m, n, o represents Gn8.1, AP01, Ghd7, Ghd8 and Gn1a, respectively; diamond represents eating and cooking quality related genes, in which $f, g, h, i, j$ represents $W x$, ALK, SBE3, SSIV-2 and SSIII- 1 , respectively; circle represents appearance quality related genes, in which c, d, e represents GW7, qSW5 and GS3, respectively; triangle represents rice BPH-resistance related genes, in which $a, b$ represents Bph6 and Bph9, respectively. The red fill represents the dominant allelic fragment.
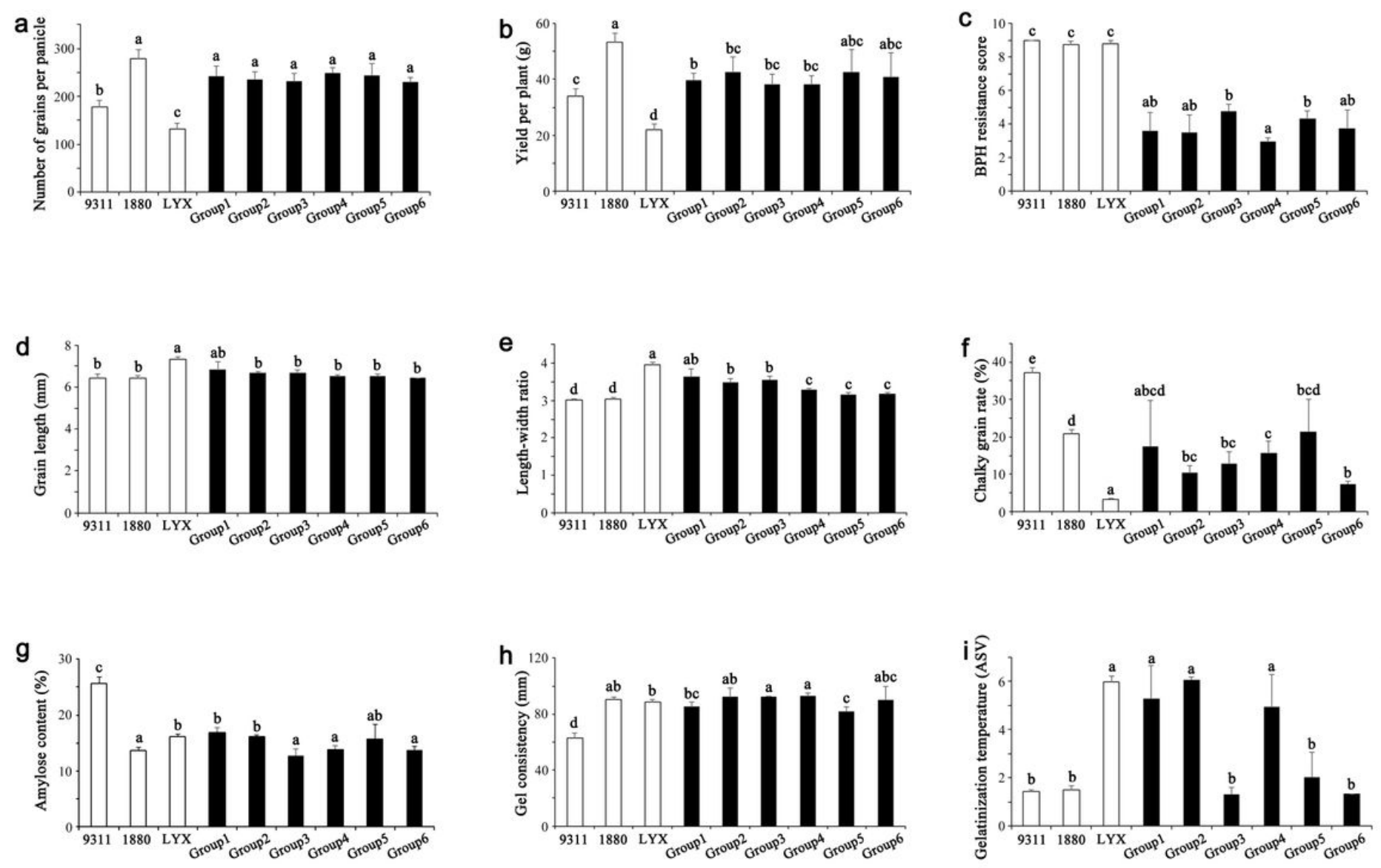

\section{Figure 3}

Phenotypic differences in groups with different target gene combinations. a: Number of grains per panicle; b: Yield per plant (g); c: BPH-resistance; d: Grain length $(\mathrm{mm})$; e: Length-width ratio; f: Chalky grain rate (\%); g: Amylose content (\%); h: Gel consistency ( $\mathrm{mm})$; i: Gelatinization temperature, which is represented by the alkali spreading value (ASV). Values are the means \pm s.d.. Letters indicate a significant difference at the $5 \%$ significance level by the least significant difference test. 
a

\begin{tabular}{|c|c|}
\hline Gene & 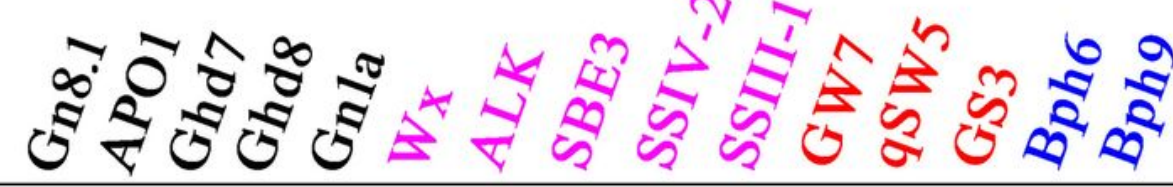 \\
\hline MD1 & A A A A A A A a A A A A A A A \\
\hline MD2 & A A A A A A A a A \\
\hline MD3 & \\
\hline
\end{tabular}

b

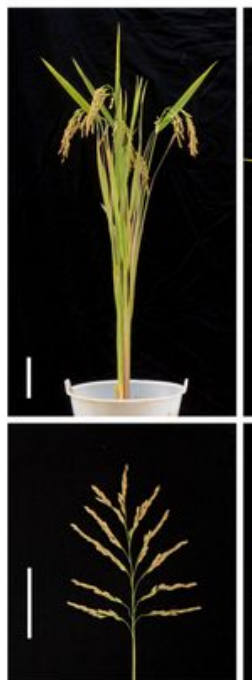

9311

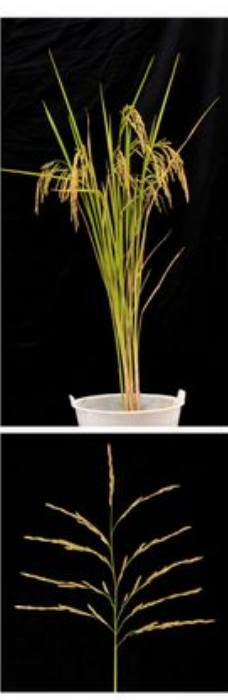

LYX

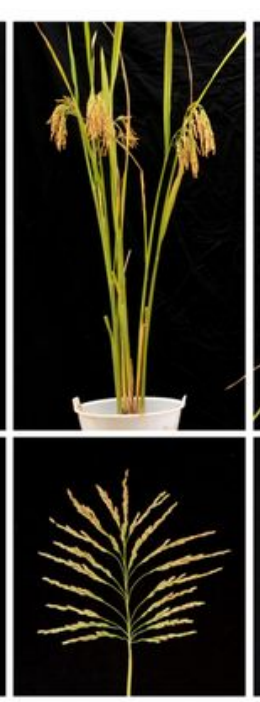

1880

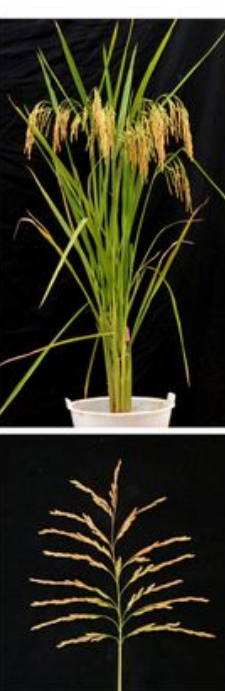

MD1

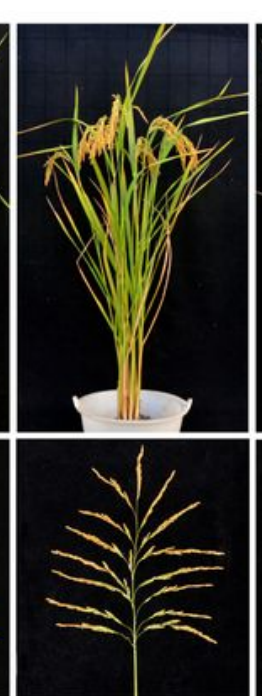

MD2

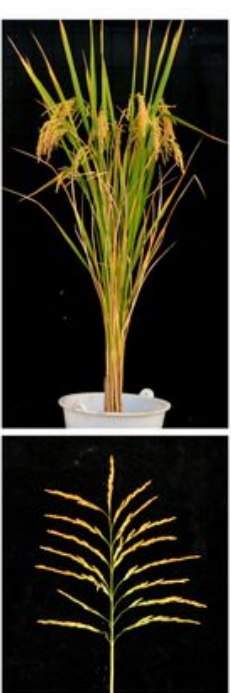

MD3

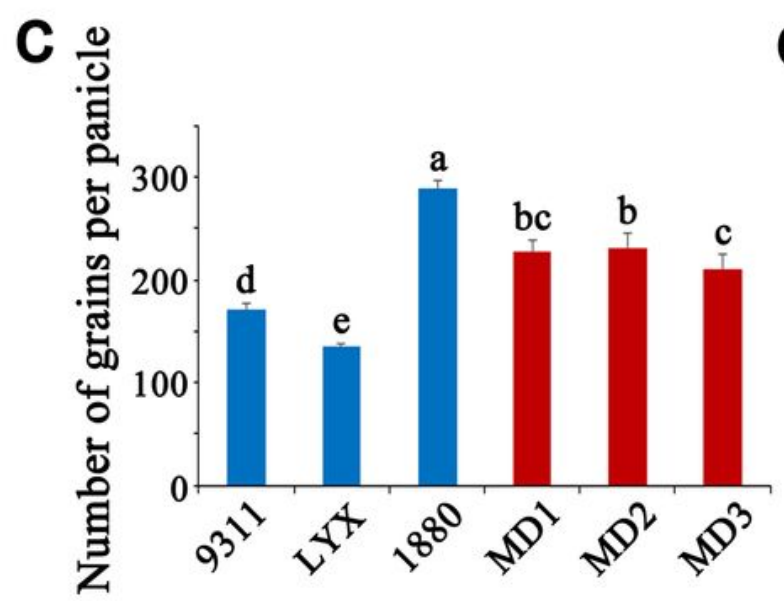

d

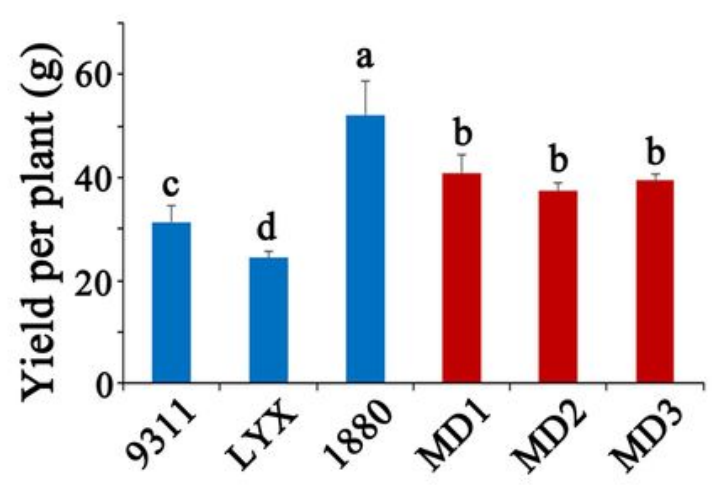

Figure 4

Gene distribution and yield performance of molecular design breeding lines. a: Genotype of the target genes in MD1, MD2 and MD3. Black represents yield-related genes, pink represents eating and cooking quality related genes, red represents appearance quality related genes, bule represents rice BPHresistance related genes. Capital letters represent dominant allelic segments. b: Gross plant and panicle morphologies. Scale bars, $10 \mathrm{~cm}$. c: Number of grains per panicle. d: Yield per plant. Values are the 
means \pm s.d., $n=15$. Letters indicate a significant difference at the $5 \%$ significance level by the least significant difference test.
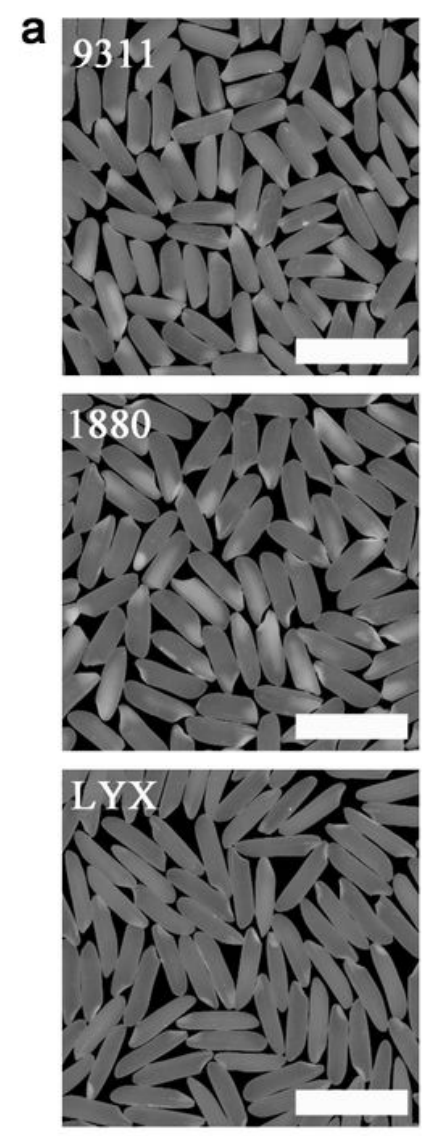
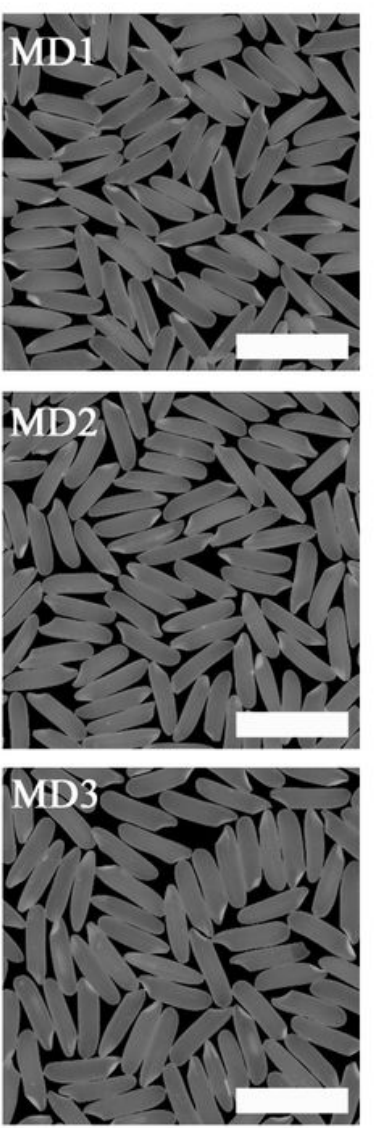
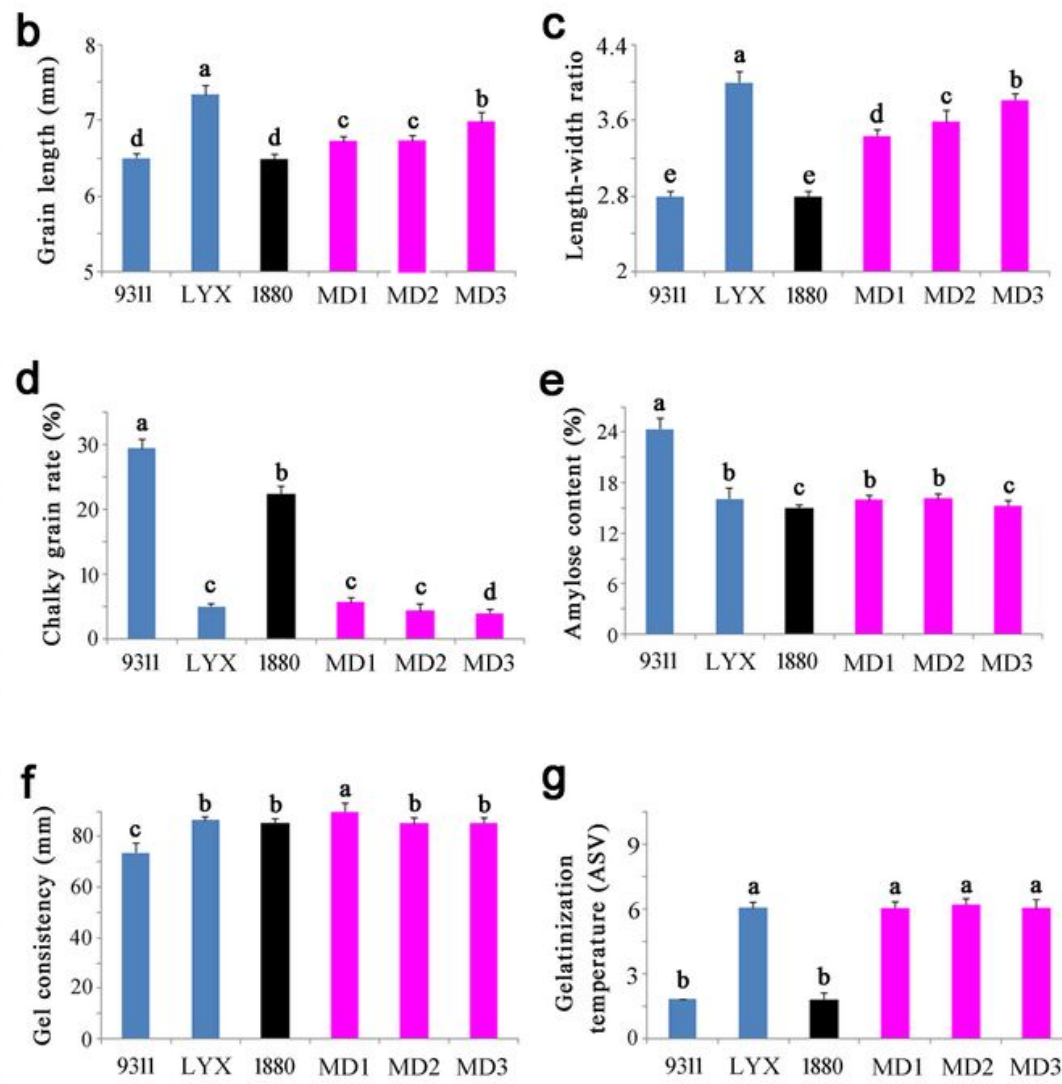

g

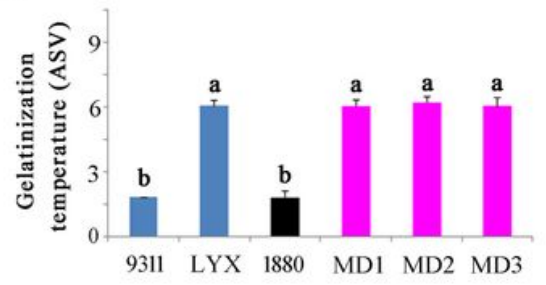

Figure 5

Rice quality performance of molecular design breeding lines and their parents. a: The performance of the milled grain. Scale bar, $1 \mathrm{~cm}$. b: Grain length. c: Length/width ratio. d: Chalky grain rate. e: Amylose content. f: Gel consistency. g: Gelatinization temperature, which is represented by the alkali spreading value (ASV). Values are the means \pm s.d., $n=15$. Letters indicate a significant difference at the $5 \%$ significance level by the least significant difference test. 

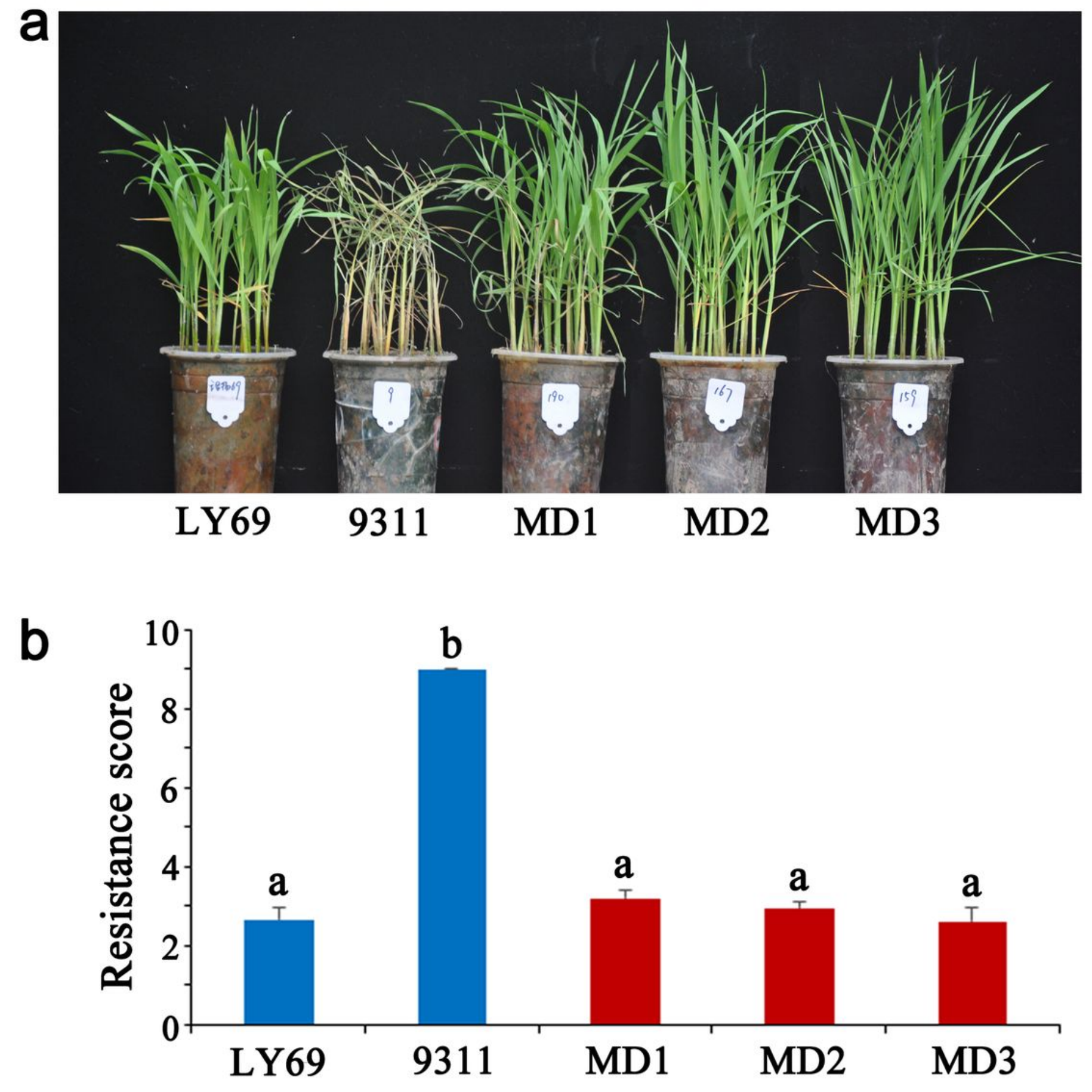

Figure 6

Resistance of brown planthopper in the molecular design breeding lines. a: Morphology of rice seedlings fed by brown planthopper. b: Brown planthopper resistance score. 9311 used as susceptible control, LY69 used as resistant control. Values are the means \pm s.d., $n=30$. Letters indicate a significant difference at the $5 \%$ significance level by the least significant difference test.

\section{Supplementary Files}


This is a list of supplementary files associated with this preprint. Click to download.

- Additionalfile1.docx

- Additionalfile2.docx 